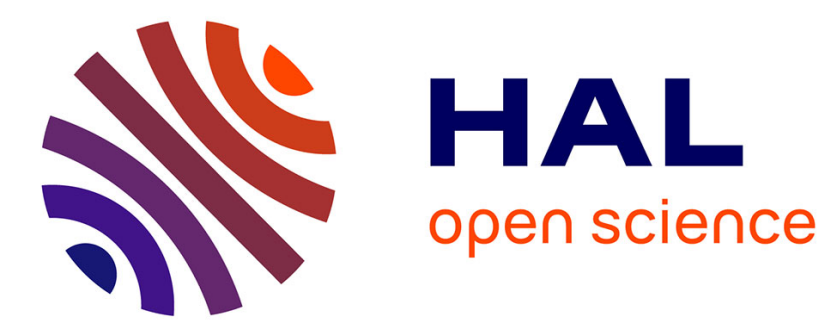

\title{
Paternités contemporaines et nouvelles trajectoires familiales
}

\author{
Agnes Martial
}

\section{To cite this version:}

Agnes Martial. Paternités contemporaines et nouvelles trajectoires familiales. Ethnologie française, 2012, XLII (1), pp.105-116. 10.3917/ethn.121.0105 . halshs-00670856

\section{HAL Id: halshs-00670856 \\ https://shs.hal.science/halshs-00670856}

Submitted on 16 Jul 2019

HAL is a multi-disciplinary open access archive for the deposit and dissemination of scientific research documents, whether they are published or not. The documents may come from teaching and research institutions in France or abroad, or from public or private research centers.
L'archive ouverte pluridisciplinaire HAL, est destinée au dépôt et à la diffusion de documents scientifiques de niveau recherche, publiés ou non, émanant des établissements d'enseignement et de recherche français ou étrangers, des laboratoires publics ou privés. 
Martial Agnès, « Paternités contemporaines et nouvelles trajectoires familiales », Ethnologie française, 2012/1 Vol. 42, p. 105-116.

Dans la majorité des pays occidentaux, les transitions familiales contemporaines ont conduit au développement et à la banalisation de situations où les hommes sont amenés à exercer leur paternité alors qu'ils ne vivent pas ou plus avec la mère de leurs enfants. Ainsi formulé, ce constat paraît relever de l'évidence. Il est cependant indissociable d'un ensemble de questions sociales, économiques et politiques ouvrant autour de la paternité, domaine d'investigation privilégié des sciences psychologiques, un axe peu exploré par les sciences sociales. L'étude socio-anthropologique des normes, des pratiques et des représentations permettant de dessiner l'évolution du rôle et du statut des pères dans nos sociétés demeure en effet, en France, relativement discrète, les recherches existantes l'éclairant principalement à travers l'étude des rôles et des statuts occupés dans la sphère intime et familiale et dans ses interrelations avec le monde du travail. Les nouvelles formes de vie familiales offrent pourtant un lieu fécond pour l'étude des transitions à l'oeuvre au sein de la paternité. Les changements dont elles témoignent s'inscrivent dans un ample processus, relevant à la fois de la redéfinition des rapports de genre et de l'évolution du contenu juridique et social des relations entre pères et enfants.

\section{Un contexte nouveau : principe d'égalité des sexes et "pluralisation »des fondements de la relation paternelle}

\section{De la hiérarchie à l'égalité au sein du couple et de la famille}

L'histoire de la paternité, à partir de la fin XIXe siècle, retrace la lente érosion d'un modèle juridique alliant hiérarchisation des sexes et inégalité des statuts liés à la filiation, au profit d'un idéal dominé par le principe d'égalité entre hommes et femmes, pères et mères, comme entre leurs enfants. La redéfinition de la paternité s'inscrit ainsi dans le passage d'une conception profondément asymétrique des rapports de genre au sein du couple et de la famille à celle d'un « couple duo » fondé en principe sur l'égalité [Théry, 2007], au fil d'une évolution scandée par l'abolition de la puissance paternelle et maritale, la dualisation de la notion de chef de famille, jusqu'à l'éclosion de la notion contemporaine de coparentalité. En France, la loi du

4 mars 2002 est venue achever une longue évolution, en attribuant aux deux parents mariés ou ayant reconnu un enfant, qu'ils cohabitent ou non, une autorité parentale conjointe que n'affecte pas la séparation, et en instaurant grâce à la résidence alternée la possibilité d'une cohabitation quotidienne de l'enfant avec ses deux parents [Neyrand, 2009]. Père et mère ont aujourd'hui, dans le mariage et hors de celui-ci, durant leur vie commune mais aussi au-delà du couple, les mêmes droits et devoirs du point de vue de la filiation et de l'exercice de l'autorité parentale ${ }^{1}$. La paternité doit trouver sa place dans ce nouvel agencement des relations et des statuts, tant du point de vue de la division des droits et des devoirs parentaux, que de la prise en charge des tâches relatives à l'éducation des enfants. Dans une société où plus de la moitié des enfants

\footnotetext{
${ }^{1}$ L'article 372 du Code civil indique ainsi que « les père et mère exercent en commun l'autorité parentale», et l'article 373-2 précise que « la séparation des parents est sans incidence sur les règles de dévolution de l'exercice de l'autorité parentale », cf. loi no 2002-305 du 4 mars 2002 relative à l'autorité parentale.
} 
naissent de parents non mariés (52,9\% en $2009^{2}$ ), l'unification progressive des droits attachés à la filiation a par ailleurs profondément transformé la relation paternelle. Cette évolution signe tout d'abord la fin de l'asymétrie entre paternité et maternité face à la «vérité » de la procréation, dès lors que le père peut être recherché en tant que géniteur. Elle contribue ensuite à donner un nouveau sens à la paternité, qui ne s'ancre plus dans « un modèle purement institutionnel» garant de la légitimité des enfants, et réfère à un modèle plus « identitaire », dans lequel chacun cherche à voir reconnaître sa "véritable » filiation [Brunet,2011].

\section{Une paternité plurielle dans ses fondements juridiques...}

Dans ce nouveau contexte s'affrontent différentes interprétations de ce qui peut fonder la relation paternelle. La volonté, que traduit la reconnaissance d'un enfant ou le consentement au mariage ? Les faits de la procréation - le père étant alors identifié au géniteur ? La réalité sociale, affective et éducative, des liens noués entre un homme et un enfant ? L'évolution juridique récente a conduit à la fragmentation de ces différentes composantes, autrefois réunies - ou présumées telles - dans le mariage. Si l'on observe aujourd'hui un recours croissant aux expertises hématologiques et génétiques en cas de conflits portant sur la filiation paternelle, diverses limites ont été apposées à la force du fait biologique (délais relatifs à la possibilité de contester une filiation, renouveau, en droit français, de la notion de possession d'état), qui traduisent la valorisation de la dimension volontaire, affective et sociale des liens père-enfant [Martial, 2008 ; Salazar, 2009 ; Brunet, à paraître]. On voit donc, dans un même mouvement, grandir la force de la référence à la " nature » et se développer l'appréhension de la réalité sociale des relations entre père et enfant, deux pôles entre lesquels vacille une définition fort incertaine du lien paternel.

\section{... et dans les nouvelles formes de vie familiales}

À cette dissociation des éléments juridiques composant la paternité fait écho l'évolution des modes de procréation et d'accès à la parentalité. Sous les auspices du " démariage », la redéfinition des trajectoires conjugales (baisse de la nuptialité, hausse des divorces et des ruptures d'union, croissance constante des familles monoparentales et recomposées) a conduit à la dissociation de l'alliance et de la filiation, de la conjugalité et des relations parents-enfants [Théry, 1993]. Familles recomposées, adoptives, ou nées du recours à la procréation médicalement assistée, hétéro ou homoparentales, voient ainsi se multiplier les relations et les statuts résultant de la conception et de l'éducation d'un enfant. Les études empiriques portant sur les représentations de la parenté dans ces différentes configurations familiales y observent la coexistence de deux grands registres métaphorique et symbolique, l'un référant aux circonstances matérielles de la conception (sperme, gamètes, gènes, sang partagé), tandis que l'autre s'appuie sur la volonté, la réalité affective et éducative, l'expérience vécue d'une relation parentale [Fine et Martial, 2010]. Cette seconde dimension revêt une importance nouvelle à travers différentes figures paternelles : celle du père adoptif et du père d'intention dans l'assistance médicale à la procréation avec donneur, ou celle du beau-

2 http://www.ined.fr/fr/pop_chiffres/france/naissances_fecondite/naissances_hors_mariage/, consulté le 6 janvier 2011. 
père dans les familles recomposées, amené à partager quotidiennement l'existence et l'éducation du ou des enfant(s) de sa compagne. Un certain nombre d'études se sont penchées sur la construction et l'évolution de ces relations, liens de parentalité « sociale » tissés dans le partage du quotidien, parfois dans le temps long de l'enfance [Théry et Dhavernas, 1991 ; Blöss, 1996 ; Cadolle, 2000 ; Martial, 2003 ; Weber, 2005]. En l'absence de reconnaissance légale des liens "beaux-parentaux », certains parcours familiaux mènent à l'adoption simple d'un enfant par son beau-père, démarche fréquemment motivée par la volonté de transmettre [Martial 2000, 2009a].

Par comparaison avec la beau-parenté, les relations unissant père et enfant dans les trajectoires post-rupture ont été moins étudiées en tant que telles. Au coeur des incertitudes entourant la définition sociale, culturelle et juridique de la filiation paternelle, les trajectoires biographiques et familiales des pères ayant connu divorces ou séparations offrent pourtant un fécond lieu d'analyse.

L'équipe réunie autour du programme de recherche "Paternités ${ }^{3}$ a ainsi formulé ses interrogations autour d'une figure singulière : le père " en solitaire », dont les liens aux enfants doivent se dispenser de la médiation quotidienne de la mère, et se jouent dans un espace et un temps redéfinis par la rupture du couple et le réaménagement des relations parentales. Ainsi énoncée, la paternité " en solitaire » recouvre une grande variété de situations, des pères " absents », qui ont vu se rompre leurs relations avec leurs enfants, à ceux - fort peu nombreux - qui assument quotidiennement l'éducation de ces derniers après la séparation ou le divorce. Entre ces deux extrêmes, les hommes réinventent leur paternité au sein d'une nouvelle organisation spatiale et temporelle, fondée sur le principe du droit de visite et d'hébergement ou sur la résidence alternée de l'enfant. À travers ces différentes situations, c'est à la réalité sociale, éducative et affective d'une paternité " en exercice » que s'intéresse la présente recherche. À partir des premiers résultats d'une enquête de terrain actuellement en cours, nous tenterons ici de dresser une trame pertinente pour l'appréhension de ces expériences

paternelles, autour de trois grands axes : l'espace et le temps qui leur sont dévolus, les relations qui les constituent, et la variabilité sociale des usages et représentations qui leur font écho.

\section{Pères et enfants dans l'après-divorce ou séparation : une relation mise à l'épreuve}

En 2009, 44,7 \% des mariages se sont soldés par un divorce en France, chiffre auquel il faut ajouter les ruptures de Pacs et d'unions libres. $57 \%$ de ces divorces impliquaient des enfants mineurs [Prioux, Mazuy et Barbiery, 2010 : 438, 440]. Les relations paternelles sont fortement interrogées et mises à l'épreuve dans les situations familiales résultant de ces désunions. Les études observent en effet, depuis la fin des années 1980, l'affaiblissement, voire la rupture des liens père/enfants dans certaines situations, et l'augmentation du nombre d'enfants « privés » ou « manquant » de père, quand bien même ce dernier existe légalement. Peu de données nouvelles permettent actuellement de connaître en France l'état des relations entre les pères et leurs enfants mineurs dans l'après-rupture. En 1994, 15 \% de l'ensemble des enfants en France ne vivaient pas avec leur père et parmi ces enfants, un sur trois ne le voyait jamais [Villeneuve-Gokalp, 1999]. Les recherches les plus récentes portent sur les relations parents/enfants à l'âge adulte

\footnotetext{
${ }^{3}$ ANR-08-JCJC-0057-01-PATERNITES. Pères en solitaires : paternités contemporaines et nouvelles trajectoires familiales (France, Espagne).
} 
et montrent que la séparation ou le divorce des parents fragilisent davantage les relations au père [Régnier-Loilier, 2006 ; Vivas, 2007].

Durant les années 1990 et 2000, une floraison de recherches nord-américaines ont étudié les motifs du « désengagement » des pères après le divorce et ses effets

sur le bien-être, le bon développement et l'intégration sociale des enfants, témoignant de l'inquiétude sociale et politique qu'a suscité la "fragilisation » de la paternité [Blankenhorn, 1995]. Celle-ci apparaît marquée non seulement par la moindre fréquence des relations paternelles, mais aussi par une participation insuffisante à l'entretien de l'enfant, et par le contenu peu éducatif de relations dans lesquelles les hommes semblent délaisser leur rôle parental [parmi les très nombreux travaux anglosaxons traitant de la question voir par exemple Kruk, 1993 ; Arrendel, 1995 ; Marsiglio, Amato et Day, 2000 ; Leite et Mc Kenry, 2002 ; Freeman, 2002]. Ainsi, le statut et le rôle des pères séparés ou divorcés ont le plus souvent été analysés en référence aux notions d'éloignement, d'absence, et de moindre investissement dans la relation aux enfants, suscitant du point de vue législatif et politique différentes tentatives de conforter les relations père-enfants [Hobson, 2002]. Cela n'a pas empêché l'éclosion de différents mouvements militants de défense des droits des pères, en Europe comme en Amérique du Nord, qui revendiquent l'application par la loi d'une égalité parentale absolue dans l'après-rupture, même si différents travaux y ont souligné quelques écarts entre discours militants et pratiques parentales [Arrendel, 1995 ; Bertoia et Drakich, 1993 ; Collier et Sheldon, 2006].

\section{Pères séparés et " nouveaux pères » : asymétries des rôles de genre et devenir paternel après les ruptures conjugales}

La fragilité de la place occupée par les hommes auprès de leurs enfants dans l'aprèsdivorce ou l'après-séparation paraît d'autant plus grande qu'elle contredit un modèle relativement récent, largement décrit par la psychologie et la sociologie de la famille, qui place les relations père-enfants sous les auspices d'une plus grande proximité. La figure traditionnelle d'un père exerçant une autorité distante à l'égard de sa progéniture tout en assumant le rôle de principal pourvoyeur du foyer a fait place à l'image d'une paternité différente, incarnée par des hommes partageant avec la mère de leurs enfants leurs anciennes prérogatives de "chef de famille » et leur statut de pourvoyeur [Neyrand, 2000 ; Dermott, 2008]. Du fait d'un partage plus égalitaire des rôles parentaux, de nouveaux usages caractériseraient la paternité contemporaine, portée par un ensemble de gestes et de paroles témoignant de l'investissement du père dans les soins et l'éducation de l'enfant [Castelain-Meunier, 2002, 2006]. Redéfinie comme une relation intersubjective, caractérisée par une forme particulière d'échange entre père et enfant, une « intimité » nouvelle faite de proximité physique, d'attachement affectif et de complicité, la paternité occupe une part croissante dans la valorisation des identités masculines contemporaines [Dermott, 2008]. Il faut bien sûr nuancer la portée du mouvement d'égalité et d'indistinction des statuts parentaux que traduirait la figure des " nouveaux pères ». Les recherches singularisent ainsi les formes d'investissement paternel, par comparaison avec la maternité, comme reposant sur des temporalités différentes, plus occasionnelles et plus fréquemment tournées vers des activités récréatives et gratifiantes, la responsabilité de l'univers domestique et familial demeurant majoritairement du ressort des femmes [voir par exemple Fagnani, Letablier, 2003 ; Bessin, Gaudart, 2009 ; Dermott, 2008 ; Doucet, 2006]. En dépit de l'accès massif des femmes à l'emploi salarié, les pères demeurent confrontés à " une définition 
hégémonique de la masculinité qui repose encore largement sur l'assignation des hommes au travail professionnel »[Connell, 1999 ; Merla, 2007 : 23], la figure du pourvoyeur conservant une place importante dans les définitions contemporaines de la paternité. En témoigne la difficulté des hommes à s'approprier les rares mesures de politiques familiales censées favoriser leur implication dans la sphère privée (congé de paternité, congé parental ou aménagements d'horaires). La paternité fait enfin l'objet d'une injonction paradoxale, opposant au modèle du père " investi », la faible reconnaissance sociale des expériences de forte implication paternelle dans l'éducation des enfants [Merla, 2007]. De cet écart entre modèle prescriptif et pratiques réelles ressort une " paternité d'intention » confrontée à la difficile remise en cause " des modalités "traditionnelles" de construction des places et des identités masculines et féminines » [Boyer et Céroux, 2010 : 57]. Les ruptures conjugales constituent à cet égard un puissant révélateur. Après la séparation, la répartition sexuée de la prise en charge des enfants semble maintenue, voire accentuée, laissant à la mère la plus grande part des prérogatives et des contraintes parentales, si bien que les premières recherches françaises portant sur les rapports de genre au sein des familles recomposées évoquaient une forme de "monoparentalité éducative » produite par une forte tendance à la « matricentralité » [Blöss, 1996 ; Cadolle, 2000]. C'est dans les milieux les moins favorisés que les pères semblent répondre le moins souvent aux normes contemporaines de la «bonne » paternité. Les identités sexuées y feraient l'objet d'un " surcodage social », opérant une distinction des rôles de sexe plus traditionnelle, dans la sphère privée comme dans l'espace public [Neyrand, $2000: 250$; Schwartz, 1990]. Les attributs associés à la paternité (autorité, domination), les formes de socialisation

liées à la relation père-enfant (liées à la transmission des savoir-faire professionnels plutôt qu'à l'univers domestique), l'expression (ténue) des valeurs et des émotions qui lui sont attachées seraient peu compatibles avec les normes contemporaines de la " bonne » relation paternelle [Jamoulle, 2005]. Dans un contexte de crise de l'emploi, la perte du statut de travailleur contribue par ailleurs à fragiliser des hommes qui ne sont plus en mesure de fournir une sécurité économique à leur famille : l'État s'est substitué à eux, devenant un pourvoyeur plus sûr à travers la distribution des prestations et minima sociaux [Jamoulle, 2005]. Après les ruptures conjugales, c'est dans les milieux modestes que la pension alimentaire est le moins souvent payée, et que l'on constate le plus fréquemment l'effacement progressif des liens des enfants à leur père [VilleneuveGokalp, 1994 ; Martin, 1997]. On doit alors y interroger la pertinence d'une norme de coparentalité soutenue par le principe d'égalité et d'indistinction de sexe [Jamoulle, 2005]. La question des rapports de genre s'avère ainsi fondamentale pour la compréhension des relations père enfants et leur évolution. Comme on vient de le voir, elle a conduit nombre d'études à porter une attention soutenue, dans les couples unis puis après la séparation, à la question de la division sexuelle du «travail parental», entendu comme un ensemble de tâches dédiées à l'éducation des enfants, au sein de la sphère domestique et dans les espaces sociaux qui leur sont associés, et comme charge mentale reposant notablement sur les femmes [Verjus et Vogel, 2009 ; Martial, 2009b]. Or, l'étude des situations de paternité " en solitaire » donne à voir des hommes expérimentant une forme nouvelle de relations à l'enfant, variant selon les modes d'organisation résidentielle qui succèdent à la rupture.

\section{L'enquête : des profils de pères peu ordinaires}

L'enquête actuellement en cours (15 entretiens ont été réalisés, 18 sont encore à venir) 
tente de saisir des trajectoires paternelles décrites par les hommes eux-mêmes, au fil d'entretiens retraçant leur histoire. Conduite d'une part auprès d'hommes ayant expérimenté diverses formes d'organisation résidentielles (droit de visite et d'hébergement, résidence alternée) grâce à la classique méthode de la «boule de neige », elle a permis d'autre part, grâce à l'aide de la Caisse d'allocations familiales des Bouchesdu-Rhône, de rencontrer des hommes se déclarant comme pères séparés ou divorcés élevant leurs enfants soit la moitié du temps (résidence alternée), soit au quotidien (résidence habituelle des enfants au domicile paternel). Ce dispositif particulier nous a amenés à rencontrer des hommes d'origines sociale et culturelle très diverses (nés à Marseille, originaires d'autres régions françaises, migrants d'origine étrangère), appartenant à des catégories socioprofessionnelles variées (RSA, invalidité, employés ou cadres du secteur public ou privé, indépendants). En outre, des types d'organisation résidentielle post-rupture a priori peu répandus y sont très représentés.

D'après le rapport de Laure Chaussebourg et Dominique Baux, fondé sur l'exploitation de 2300 jugements de divorces et 1402 ordonnances concernant des enfants de parents séparés rendus en 2003, l'attribution de la "résidence habituelle » au domicile maternel demeure majoritaire (79\% des divorces, $84 \%$ des séparations). Bien que minoritaire $(11,5 \%$ des divorces, $6 \%$ des séparations), la résidence alternée s'est installée dans les usages. Un petit nombre d'hommes élèvent enfin quotidiennement leurs enfants après la rupture conjugale (7\% des divorces, $8 \%$ des séparations) [Chaussebourg et Baux, 2007 : 4, 7]. Parmi les pères que nous avons rencontrés, six ont fait l'expérience du droit de visite et d'hébergement, quatre ont pratiqué ou pratiquent aujourd'hui la résidence alternée, et neuf vivent quotidiennement seuls avec leurs enfants ${ }^{4}$. Certes peu représentatives au regard de l'ensemble des situations d'aprèsdivorce ou séparation, ces deux dernières situations sont encore mal connues, la plupart des études traitant généralement de trajectoires paternelles plus classiques associées au droit de visite et d'hébergement du père. Cette enquête est ainsi l'occasion d'explorer et de mettre en regard des formes de paternités très différentes, que l'on peut considérer comme " exemplaires ", non pas du fait de leur nombre, mais parce qu'elles amènent à réinterroger les clivages traversant les rapports de genre et les différences sociales dans la mise en acte des statuts parentaux. Cette première exploration a pour objet de mettre en évidence cette diversité, et de faire émerger, dans les nouvelles trajectoires familiales, des dimensions peu étudiées de la paternité.

\section{Des pères « intérimaires »}

Comme nous le suggérons avec Agnès Fine dans un autre article de ce numéro, l'organisation du droit de visite et d'hébergement instaure une relation parentale discontinue, éprouvée par l'absence de corésidence et de temps quotidien. L'attribution massive de la résidence habituelle des enfants au domicile maternel a suscité diverses analyses quant au rôle joué par l'institution judiciaire au moment des divorces et des séparations. S'il arrive qu'elle soit imposée par la justice à des pères désireux d'assumer quotidiennement l'éducation de l'enfant, surtout lorsque celui est jeune, ces situations

\footnotetext{
${ }^{4}$ Différents modes de résidence peuvent se succéder au cours d'une même trajectoire : certains pères s'étaient vu octroyer un droit de visite et d'hébergement ou avaient décidé, en accord avec la mère des enfants, d'une résidence alternée, avant que les enfants ne viennent vivre avec eux à temps plein. C'est pourquoi nous trouvons plus de modes de résidences que de pères.
} 
semblent minoritaires, le choix du domicile maternel comme résidence principale de l'enfant étant dominant dans les procédures de divorce par consentement mutuel, dans lesquelles le juge entérine les décisions parentales [Bertaux et Delcroix, 1991 ; Théry, 1993 ; Bessière et al., 2010]. La rupture inaugure alors une phase où le temps et l'espace cessent d'être quotidiennement partagés. «Quand on part, on pige pas tout de suite que c'est la dernière fois qu'on est chez nous et qu'on voit ses enfants tout le temps et tout. On comprend pas tout de suite ça, et puis il y a quand même un retour assez rapide. C'est une impression, une phase de déchirement [...] Une espèce d'exil » (Bernard, 41 ans, informaticien, deux enfants).

Le droit de visite et d'hébergement signe ainsi la fin d'une relation inscrite dans la corésidence quotidienne, élément fondamental de la constitution et l'activation des relations entre parents, et lieu « habituel » des relations parentales, telles que les conçoit aujourd'hui notre société. " C'est le drame de cette séparation, de perdre mon quotidien avec mes enfants. Ça, ça a été dur, parce que quand je les voyais le mercredi, je les voyais pas le week-end, et je les revoyais que le mercredi suivant, une semaine sans les voir, c'était très récurrent [...] J'étais absolument heureux quand ils

étaient là » (Jean, 59 ans, universitaire, deux enfants). Esther Dermott [2008] remarque, dans son analyse de la paternité contemporaine, que la question du temps est différemment abordée par les pères en couple et par les pères devenus "non résidents ». Si les premiers ne font pas du temps une question centrale dans leur relation à l'enfant ceci justifiant implicitement qu'ils se rendent moins disponibles que leur compagne -, les seconds insistent sur la signification d'une temporalité désormais comptée, exprimant le sentiment d'une dépossession, d'une perte de contrôle de leur relation aux enfants[Dulac, 1995 ; Bradshaw et al., 1999 ; Trinder et al., 2002]. L'absence de quotidien partagé ébranle en outre l'évidence, la spontanéité et l'intimité des relations paternelles, désormais contenues tout entières dans les « visites » de l'enfant, qu'il faut à tout prix réussir. Patrick (53 ans, conseiller en entreprise, deux enfants) souligne ainsi la valeur plus grande qu'il donne désormais aux moments passés avec ses enfants, en vertu d'un droit de visite et d'hébergement « classique » (un week-end sur deux et la moitié des vacances scolaires) élargi cependant à deux soirs par semaine. " Je me suis rendu compte que le temps du coup que j'avais avec les enfants, il était super précieux, par rapport au temps que j'avais avant qui était normal. Donc du coup tu valorises un peu le temps passé d'une façon différente, tu le juges de façon différente. » Patrick continue à beaucoup s'occuper de ses enfants, c'est-à-dire à partager avec eux, comme avant, diverses activités de loisirs et des moments privilégiés, le week-end ou certains soirs de semaine. Mais c'est la mère des enfants qui- comme avant, semble-t-il - gère tous les éléments relatifs à la vie des enfants (scolarité, santé, vêtements, logistique). Patrick, alors qu'il est objectivement souvent présent, apparaît comme occupant un rôle secondaire à leur égard. Certains pères se vivent ainsi comme des " parents de second ordre », réduits au rôle d' « oncle en visite », ou de "parent éloigné » [Arrendel, 1995 ; Queniard, 1999], la mère occupant le rôle principal. Ils s'estiment parfois assignés à un statut de simple pourvoyeur, puisqu'ils doivent désormais s'acquitter d'une pension alimentaire sans bénéficier de relations quotidiennes avec leurs enfants. Dans ces conditions, de nombreux travaux ont souligné la fragilité des relations père-enfants, accentuée par la difficulté des hommes à délier leur paternité de l'ancienne relation conjugale. Les liens aux enfants peuvent en effet, à l'instar des relations à l'ex-compagne, devenir transitoires [Arrendel, 1995 ; Cadolle, 2000]. De plus, les conflits et tensions accompagnant la rupture, la réticence éventuelle de la mère à la poursuite de relations paternelles tendent aussi parfois à décourager les hommes, qui laissent alors 
progressivement se défaire les liens [Quéniart, 1999]. L'entrelacs complexe des sentiments amoureux et parentaux traverse nombre de récits paternels. Ainsi Franck (47 ans, indépendant, trois enfants dont un né d'une première union) explique en décrivant les deux années qui ont suivi la séparation d'avec la mère de son fils : «J'avais peur de confondre la détresse dans laquelle j'étais avec l'amour pour mon fils. Que les deux s'interpénètrent et que l'énergie que je mettais à entretenir la relation avec mon fils, elle soit empreinte de la détresse de la séparation. "S'ils correspondent fréquemment à une phase confuse et douloureuse de la vie des pères, ces moments sont aussi décrits comme une forme de mise à l'épreuve et de reconstruction de soi. Franck évoque ainsi des relations très difficiles avec la mère de son fils, qui l'a quitté pour un autre homme, alors que la famille était partie vivre à l'étranger. Il décrit deux années chaotiques, où se mêlent la douleur de la séparation conjugale et la crainte de perdre contact avec l'enfant, alors tout petit, dans un contexte de grande fragilité sociale, matérielle et juridique (il n'a pas l'autorité parentale), loin du pays d'origine ; il raconte ensuite le retour en France de son ex-compagne et de son fils, qu'il rejoint au bout de deux mois, et les négociations qui mèneront à un accord sur le partage de l'autorité parentale, ainsi qu'à l'organisation d'une forme de résidence alternée.

Il reprend alors des études, ouvre un cabinet d'architecture, rencontre une nouvelle compagne avec qui il a deux enfants. Dans le récit de Franck, sa capacité à demeurer père en dépit de la séparation est un enjeu majeur, qui légitime et accompagne ses choix de vie, sa trajectoire professionnelle, et la suite de son histoire familiale. S'il est essentiel d'interroger ce trait caractéristique de la paternité post-rupture qu'est la séparation, l'absence de temps et d'espace quotidien, il importe par ailleurs de ne pas la réduire à ce " manque ", et d'observer les stratégies dont font preuve les hommes dans la réinvention de leur relation aux enfants.

Celle-ci peut s'incarner ailleurs que dans l'espace domestique tout en existant quotidiennement, dès lors que père et enfants vivent à proximité. Ainsi Bertrand (informaticien, deux enfants), qui ne vit plus avec la mère de ses enfants, et ne les reçoit pas non plus chez lui - son appartement est trop petit -, passe cependant du temps avec eux les mercredis après-midi, et les prend chaque matin chez leur mère pour les amener à l'école.

En outre, dès lors que les enfants grandissent, d'autres expériences se dessinent, qui ont trait, par exemple, à l'apprentissage et à l'initiation. Jean(59 ans, universitaire) a ainsi emmené ses deux enfants adolescents en voyage, dans différents pays, partant toujours seul avec eux alors qu'il avait renoué une relation conjugale cohabitante. Il souligne, non sans fierté, le caractère un peu aventurier de ces voyages autour du monde, toujours vécus à trois, et dont il a conservé de précieux souvenirs. Plus prosaïquement,

Franck (47 ans, indépendant) a récemment aidé son fils Yann, âgé de 21 ans, à rénover un appartement, activité qu'il a lui-même pratiquée longtemps, et qui permettra à Yann de gagner un peu d'argent pour ses études : « On s'est fait une petite initiation [...] Lui a pris le chantier, et puis moi, je suis venu en renfort. " Franck décrit avec plaisir ce moment partagé, autour de la transmission d'un savoir-faire plutôt associé au masculin dans notre société, une activité physique et dure : "Ça te met dans la réalité. C'est plus la théorie d'un père par rapport au fils, c'est la réalité ensemble. Bon ça va, si t'es consciencieux, mais il faut surtout s'arracher, c'est dur. Et on l'a fait tous les deux, je suis super content. »

Enfin, la présence d'un environnement familial entourant le père et ses enfants, notamment lors des visites de ces derniers ou durant les vacances, tend à réinscrire la paternité au sein d'un cadre collectif rassurant, qui peut rompre l'éventuelle solitude du 
père face à ses enfants. Jean (universitaire, deux enfants) retrouvait ainsi ses parents, frère et soeur avec ses enfants chaque dimanche de visite. "Vous savez, les Arméniens, on est très famille [...] Mon père, ma mère, une maison familiale, donc on se retrouve avec ma soeur, ses enfants qui ont l'âge des miens, ils étaient très proches, mon frère, on se retrouvait en famille le dimanche, assez souvent [...] Ils sont très proches, Émilie et Fabien, de leurs cousins qui ont leur âge. »

\section{Pères à plein temps}

À l'autre extrême, dans la manière dont l'après-rupture donne à voir la paternité, on trouve des pères élevant leurs enfants seuls et au quotidien. La plupart des mères de ces enfants ont conservé des contacts avec eux, parfois de manière très régulière, en vertu d'un droit de visite et d'hébergement négocié entre parents ou décidé par le juge, d'autres de façon plus espacée et moins prévisible. Certaines d'entre elles s'étaient vu confier, lors de la rupture, la résidence habituelle, ou devaient accueillir les enfants en résidence alternée, mais cette solution n'a pas duré.

Quelles qu'en soient les raisons, la résidence au domicile paternel n'a jamais été décidée, comme ce peut être le cas dans nombre de séparations et de divorces instaurant un droit de visite et d'hébergement pour le père, sans que les compétences de l'autre parent soient mises en question.

Dans le discours de ces pères seuls, quel que soit leur milieu d'origine, le registre de la défaillance maternelle est sans cesse présent. Inconstance amoureuse et sexuelle, instabilité psychologique, difficultés économiques et matérielles, absence relative d'intérêt pour la maternité : les motifs varient, mais les pères s'accordent à constater que les mères de leurs enfants ne sont pas de "bonnes » mères. Ce discours négatif témoigne avant tout de la puissance des normes définissant la maternité et les conduites qui lui sont associées dans nos sociétés contemporaines. Il retentit également dans l'inquiétude que ces hommes expriment fréquemment au sujet du bien-être de leurs enfants, craignant de ne pouvoir compenser l'éloignement de la mère.

Ainsi ces pères au quotidien ne remettent- ils pas vraiment en cause " l'évidence maternelle » justifiant dans la majorité des cas la résidence au domicile de la mère [Bessière et al., 2010]. Ils pallient son absence, de manière plus ou moins contrainte. Mais ces paternités quotidiennes et solitaires n'en transforment pas moins les pères et leurs relations aux enfants. Le temps et l'espace de la paternité sont en effet métamorphosés par la rupture : ils sont désormais saturés d'enfants, de travail domestique et de tâches éducatives, qu'il faut concilier avec le maintien d'un emploi assurant un revenu plus ou moins élevé, et pour lesquels il faut aussi parfois renoncer à une vie sociale qui s'amenuise ou disparaît. Pour certains - ils sont rares - cette situation était installée dès avant la séparation.

Yvan (47 ans, régisseur de théâtre, deux enfants) se présente ainsi comme ayant toujours assumé la gestion quotidienne de la maisonnée. La rupture a finalement induit peu de changements dans sa vie familiale, si ce n'est la fin des tensions et des conflits conjugaux. Medhi (Algérien, 38 ans, employé en CDD dans une société de nettoyage, en France depuis dix ans) raconte qu'il a toujours été proche de son fils, par exemple durant les premiers mois de l'enfant, qu'il a veillé de longues nuits. Sa situation de père seul s'inscrit pour lui dans une relative continuité. Pour d'autres, la rupture a conduit à un véritable bouleversement. Mario, Brésilien, travaillant comme charpentier en Guyane 
française au moment de la rupture, s'est par exemple retrouvé seul avec quatre enfants en bas âge. Il décrit cette période comme la plus difficile, mais aussi la plus forte et la plus riche de sa vie : la paternité quotidienne est aujourd'hui devenue une dimension centrale et valorisante de son identité sociale. D'autres, moins positifs, portent cette monoparentalité subie et les tâches domestiques, les responsabilités parentales qui l'accompagnent comme un fardeau beaucoup trop lourd, chargé d'angoisse et d'inquiétudes éducatives, matérielles et financières. Les inégalités

socio-économiques sont ici très présentes. Concilier un emploi et la présence de jeunes enfants au domicile paternel est en effet d'autant plus difficile que les hommes sont peu qualifiés. Presque tous ont dû adapter leur vie professionnelle à ces nouvelles contraintes, ce qui semble finalement plus aisé pour les cadres et les ingénieurs que pour les petits employés, ou les hommes travaillant grâce à de petits contrats à durée déterminée, dont ils ne peuvent négocier les horaires.

Certains, à l'instar de Mario, ont renoncé à travailler, et perçoivent le RSA en attendant que les enfants grandissent. Ces inégalités caractérisent aussi l'espace, souvent trop petit, inadapté à l'accueil de fratries parfois importantes. De nombreux pères n'ont pas de chambre à eux dans le logement qu'ils occupent, et souffrent de l'inconfort des conditions de vie familiale, mais aussi d'une absence totale d'intimité. Même lorsque les conditions matérielles et économiques permettent d'aménager confortablement ces paternités en solo, la charge des enfants se fait sentir, si bien que l'espace familial devient oppressant, au point que l'on s'en échappe avec soulagement. Ivan raconte ainsi qu'il lui est arrivé de "faire le mur », une fois les enfants couchés, pour retrouver des amis et se détendre... après avoir toutefois prévenu la voisine de son départ.

L'absence d'une nouvelle histoire de couple semble représenter une dimension importante de ces formes de paternité. L'enquête induit ici un biais : les pères seuls que nous avons rencontrés sont nécessairement célibataires puisqu'ils se déclarent comme tel à la CAF au moment de l'entretien. Solitude amoureuse, célibat conjugué à quelques aventures hors de la vie familiale, ou tentatives insatisfaisantes de vie commune : il semble dans ces cas que la paternité " en solitaire " rejoigne les expériences de monoparentalité féminine. Le sentiment d'isolement lié au caractère marginal de leur situation est enfin rapporté par tous ces hommes, quel que soit leur milieu social. Divers auteurs évoquent ainsi la remise en question, par l'entourage proche et l'environnement social, de la conformité des pères impliqués dans l'éducation quotidienne des enfants aux normes définissant la masculinité (virilité, force physique, hétérosexualité). Terry Arrendel [1995] qualifie d'ailleurs les hommes divorcés investis dans le travail parental auprès de leurs enfants de pères " androgynes », parce qu'ils disent s'être approprié des comportements parentaux et des attitudes caractérisés comme féminins, évoquant notamment l'importance des soins nourriciers aux enfants, et référant à des modèles féminins dans leur environnement proche (la mère, l'ex-conjointe, la soeur). Ces pères expérimentent d'importants conflits "intra-genre », affrontant un discours réflexif négatif sur leur manière d'être et de faire avec leurs enfants, notamment de la part de l'entourage masculin. Ce manque de reconnaissance peut conduire à un sentiment d'exclusion et d'isolement à l'égard des formes de sociabilité des deux sexes, lié à la fois à la difficulté d'accéder aux réseaux féminins constitués autour de l'enfance, et à l'absence d'un groupe de pairs masculins partageant la même expérience [Merla, 2007]. La présence autour de ces hommes d'un réseau familial aidant détermine aussi la manière dont ils vivent leur paternité [Allard et al., 2005]. Elle est très inégale selon les cas. La famille paternelle peut apporter une aide pratique non négligeable, quotidienne 
ou ponctuelle, relayant le père dans la prise en charge des enfants, palliant aussi l'isolement relationnel que connaissent certains pères migrants, pour qui de rares retours au pays d'origine constituent les seuls moments de partage de la charge parentale. Au-delà de l'aide pratique, la présence de l'entourage familial réinscrit la paternité « solitaire » au sein d'un collectif où l'enfant peut situer, en même temps que son père, son appartenance et son identité.

\section{La résidence alternée : une paternité nouée dans le partage des charges parentales}

Les cas de résidence alternée sont les moins nombreux dans l'état actuel de la recherche. Alors que cette solution est la plus récente et sans doute la plus nouvelle, c'est peut-être aussi celle qui semble le moins transformer la manière dont les hommes affirment vivre leur paternité. Elle assure tout d'abord le partage d'un temps et d'un espace quotidien à des hommes qui ne concevaient pas, disent-ils, de vivre sans leurs enfants. Le choix de la résidence alternée est par ailleurs observé dans tous les milieux rencontrés, et des pères très peu qualifiés ont fait valoir très clairement qu'ils ont souhaité, dès la rupture, s'occuper au quotidien et à mi-temps de leurs enfants. Parmi ceux qui élèvent seuls leurs enfants en attendant un engagement plus grand de la mère dans la vie de ces derniers, la résidence alternée est fréquemment mentionnée comme la solution espérée : rares sont les pères "à plein temps " prêts à renoncer à toute vie quotidienne avec leurs enfants. Si l'on se fie toutefois aux études récentes portant sur le sujet, la mère continue fréquemment de jouer un rôle prépondérant dans ce type d'organisation. Les recherches récentes observent en effet, au sein des duos parentaux, des interprétations et investissements différenciés de la prise en charge des enfants, qui signent parfois la reconduction des asymétries antérieures à la séparation [Brunet, Kertudo, et Malsan, 2008 ; Cadolle, 2008]. La résidence alternée aménage en outre des moments de " pause parentale » permettant aux hommes de consacrer du temps à d'autres dimensions de leur vie. Les pères y semblent d'ailleurs plus nombreux à avoir renoué une vie conjugale cohabitante. Dans l'étude de l'après-divorce, dominé par le système du droit de visite et d'hébergement, de nombreux travaux ont souligné que la nouvelle union paternelle est corrélée à une moindre fréquence de ses relations aux enfants du premier lit ainsi qu'à un moindre soutien financier, qui se lit dans les conflits liés à l'entretien de l'enfant et lors de l'entrée des jeunes dans la vie adulte [VilleneuveGokalp, 1994 ; Martial, 2005 ; Cadolle, 2005]. Nos premiers entretiens suggèrent, dans les cas de résidence alternée, que l'arrivée d'une nouvelle conjointe, et parfois la naissance d'un nouvel enfant peuvent aussi conforter les hommes dans l'exercice de leur paternité à l'égard de l'enfant de l'union précédente. François ( 44 ans, artiste, une enfant) décrit de manière très positive la relation de sa nouvelle compagne et de sa fille, âgée de 11 ans, qu'il accueille en résidence alternée depuis qu'il est séparé de sa mère. L'arrivée de Louise a changé la donne : "Elle s'occupe beaucoup de Lola. Elle a une activité professionnelle qu'elle mène essentiellement à la maison, elle se sent mieux travailler à la maison avec son ordinateur portable et gérer le temps comme elle le veut. Et du coup, elle a tout recréé un peu [...] Déjà, elle a essayé de trouver sa place, c'était pas forcément évident parce qu'elle arrivait dans un couple : une petite fille et son papa. » Implication dans l'organisation domestique, allées et venues à l'école, suivi des devoirs mais aussi complicité et petits cadeaux (vêtements, livres) : Louise paraît très présente, et la paternité de François se conjugue avec cette nouvelle figure beaumaternelle dans une nouvelle unité familiale. Baptiste (32 ans, indépendant, deux 
enfants), père d'un petit garçon à l'âge de 25 ans, a quitté sa première conjointe trois ans plus tard, décidant avec elle d'une résidence alternée pour l'enfant. Celle-ci fonctionne toujours aujourd'hui, alors qu'il a formé un nouveau couple et donné naissance à une petite fille. Il décrit le rôle bénéfique qu'a joué sa deuxième compagne en l'aidant à équilibrer sa relation à son fils, et l'implique constamment dans son récit, chaque fois qu'il évoque l'éducation de l'enfant. Ainsi la résidence en alternance paraît-elle favoriser une paternité mise en oeuvre dans le partage des tâches et des compétences relatives à l'éducation des enfants, tant avec la mère qu'avec une nouvelle compagne. Le caractère « solitaire » de la paternité après-divorce y paraît alors à interroger...

Il se pourrait aussi que la résidence alternée, en favorisant le partage d'une vie familiale quotidienne entre la nouvelle compagne du père et les enfants, soit à même de permettre l'éclosion d'une relation beau-maternelle plus gratifiante et valorisée que dans le cadre du droit de visite et d'hébergement. Au terme de cette première exploration, nous ne pouvons encore que tracer quelques pistes propres à guider l'analyse des formes de paternité « en solitaire».

$\mathrm{Au}$ départ des interrogations fondatrices de cette recherche se trouvait un constat : après la rupture conjugale, la paternité apparaît comme déliée de la maternité et doit désormais exister hors du couple et de la cellule familiale. Dans l'exercice de cette paternité apparaît d'abord l'importance fondamentale du rapport au temps : confrontée à l'absence d'une temporalité quotidienne, comme au temps surchargé de la monoparentalité, la paternité se trouve mise à l'épreuve dans ses relations de distinction, d'équivalence et d'inégalité avec la maternité. La diversité des témoignages et des perceptions dont témoignent les récits de pères issus de milieux sociaux très différents amène alors à nuancer les clivages sociaux présumant la " rigidité » des rapports de genre au sein des milieux populaires. Traversés de tensions et de changements, ceux-ci ne sont pas un bloc monolithique et immuable. Là comme ailleurs sont observables des manières d'être et d'inventer révélatrices de formes nouvelles de paternité.

Les récits recueillis montrent aussi que la relation père-enfants (tout comme la relation mère-enfants) ne se réduit pas à la prise en charge éducative et matérielle des enfants, au sein de l'univers domestique. En tant que relation parentale mise en jeu dans les relations d'apprentissage et d'initiation, elle offre d'autres supports d'investigation et de légitimation. Il faudra également tenir compte, dans la suite de cette recherche, des enjeux de reproduction sociale qui traversent la paternité, garante

de l'accès de l'enfant à l'autonomie, via un statut et une identité sociale qui passe, aujourd'hui, par l'emploi. Céline Bessière montre par exemple comment la volonté de transmettre la terre et le métier qui l'accompagne chez les jeunes pères viticulteurs de la région de Cognac les mène parfois à demander la résidence de l'enfant au moment d'une séparation - car pour que la vocation du métier puisse éclore, il faut grandir au

plus près de la terre [Bessière, 2007]. Aurélie Fillod- Chabaud [2009] analyse, à travers les parcours de certains membres de l'association SOS Papa, comment les impératifs de reproduction sociale peuvent amener certains pères séparés de leur enfant à investir très fortement leur devenir scolaire. La paternité existe enfin au sein d'une constellation de relations affectives, juridiques et symboliques, que les études de la paternité contemporaine, centrées sur le partage des tâches au sein du couple et la relation pèreenfants, explorent peu. S'il importe de tenir compte du devenir des relations à la mère, puis d'une éventuelle recomposition familiale, la paternité s'inscrit aussi dans 
une amplitude relationnelle lisible tant dans les usages sociaux de la parenté, qui permettent de la replacer dans un cercle familial étendu, que du point de vue de la transmission d'une identité familiale [Attias-Donfut et Segalen, 1998 ; Weber, 2005]. À travers ces différentes dimensions, il s'agit de considérer la paternité " en solitaire » comme un révélateur des transitions à l'oeuvre au sein des rapports de genre, et comme une relation de parenté, régie par différentes lois, inscrite dans un ensemble relationnel qui lui donne sens, et se construisant au fil d'une temporalité à la fois quotidienne, biographique et générationnelle.

\section{Références bibliographiques}

ALLARD Francine et al., 2005, « Maintien de l'engagement paternel après une rupture conjugale : point de vue de pères vivant en contexte de pauvreté », Revue Enfances, Familles, Générations, 3, "Paternité : bilan et perspective » : consulté le 24 août 2011. URL : http : //id.erudit.org/iderudit/012537ar.

ARRENDEL Terry, 1995, Fathers and Divorce, Newbury Park, California, Sage Publications.

ATTIAS-DONFUT Claudine et Martine SEGALEN, 1998, Grands-parents : la famille à travers les générations, Paris, Odile Jacob.

BERTAUX Daniel et Catherine DELCROIX, 1991, " Des pères face au divorce : la fragilisation des liens paternels », Collection Espace et famille, 17, Paris, CNAF.

BERTOIA Carl E. et Janice DRAKICH, 1993, " The Father's Right Movement. Contradictions in Rhetoric and Practice ", Journal of Family Issues, 14, 4 : 592-615.

BESSIÈRE Céline, 2007, "Se marier pour aller jusqu'au bout ensemble ? Ruptures conjugales et transmission des exploitations agricoles dans la lignée ", Revue d'études en Agriculture et Environnement, 88, $3:$ 44-70.

BESSIÈRE Céline, Sibylle GOLLAC et al., 2010, "Au tribunal des couples. Situations professionnelles des conjoints et procédures judiciaires de séparation conjugale », Rapport de recherche pour la Mission Droit et Justice, Paris.

BESSIN Marc et GAUDART Corinne, 2009, "Les temps sexués de l'activité : la temporalité au principe du genre ? », Temporalités [En ligne], mis en ligne le 30 septembre 2009, consulté le 25 février 2011. URL : http://temporalites.revues.org/ index979.html.

BLANKENHORN David, 1995, Fatherless America. Confronting our most urgent social problem, New York, Basik Books.

BLÖSS Thierry, 1996, Éducation familiale et beau-parenté : l'empreinte des trajectoires biographiques, Paris, L'Harmattan.

BOYER Danielle et Benoît CÉROUX, 2010, " Les limites des politiques publiques de soutien à la paternité », Travail, genre et sociétés, 24 : 47-62.

BRADSHAW Jonathan et al., 1999, Absent Fathers?, London,

Routledge.

BRUNET Florence, Pauline KERTUDO et Sylvie MALSAN, 2008,

Étude sociologique de la résidence en alternance de parents séparés,

Dossier d'étude, CAF, 109 : 1-114.

BRUNET Florence, 2011, «Des usages protéiformes de la nature : essai de relecture du droit français de la filiation », in Pierre Bonte et al. (dir.), L'argument de la filiation aux fondements des sociétés européennes et méditerranéennes, éditions de la MSH : 285-323. CADOLLE Sylvie, 2000, Être parent, être beau-parent, La recomposition de la famille, 
Paris, Odile Jacob.

- 2005, " C'est quand même mon père ! La solidarité entre père divorcé, famille paternelle et enfants adultes », Terrain, 45 : 83-96.

- 2008, «La résidence alternée : ce qu'en disent les mères », Informations sociales, 149 : 68-81.

CASTELAIN-MEUNIER Christine, 2002, La place des hommes et les métamorphoses de la famille, Paris, Presses Universitaires de France.

- 2006, Les métamorphoses du masculin, Paris, Presses Universitaires

de France.

CHAUSSEBOURG Laure et Dominique BAUX, 2007, L'exercice de l'autorité parentale après le divorce ou la séparation des parents non mariés, Ministère de la Justice.

COLLIER Richard et Sally SHELDON (eds.), 2006, Fathers' Rights, Fatherhood and Law Reform in Comparative Perspective, Oxford, Portland, Hart Publishing.

CONNELL Raewyn W., 1999 [1995], Masculinities, Cambridge, Polity Press.

DERMOTT Esther, 2008, Intimate Fatherhood : A Sociological Analysis,

New York, Routledge.

DHAVERNAS Marie-José et Irène THÉRY, 1991, Le beau-parent

dans les familles recomposées. Rôle familial, statut social, statut juridique,

CNAF.

DOUCET Andrea, 2006, Do Men Mother ? Fathering, care and responsability, University of Toronto Press.

DULAC Germain, 1995, "Rupture d'union et déconstruction du lien père-enfant 》, PRISME, 5, 2-3 : 300-312.

FAGNANI Jeanne et Marie-Thérèse LETABLIER, 2003, " S'occuper des enfants au quotidien : mais que font les pères ? », Droit social, $3: 251-259$.

FILLOD-CHABAUD Aurélie, 2009, L'expérience judiciaire des pères et l'accès au droit en matière de droit de garde, mémoire de master sous la direction de Florence Weber, ENS, EHESS.

FINE Agnès et Agnès MARTIAL, 2010, «Vers une naturalisation de la filiation ? », Genèses, $1,78: 121-134$.

FREEMAN Tabitha, 2002, « Loving fathers or Deadbeat Dads : the crisis of Fatherhood in Popular Culture ", in Sarah Earle and Gayle Letherby (eds.), Gender, Identity and Reproduction : Social perspectives, London, Palgrave : 33-49.

HOBSON Barbara (ed.), 2002, Making Men into Fathers. Men, Masculinities and the Social Politics of Fatherhood, Cambridge University Press.

JAMOULLE Pascale, 2005, Des hommes sur le fil. La construction de l'identité masculine en milieux précaires, Paris, La Découverte.

KRUK Edward, 1993, Divorce and Disengagement-Patterns of Fatherhood within and beyond Marriage, Halifax, Fernwood Publishing.

LEITE Randall W. and Patrick C. MC KENRY, 2002, " Aspects of Father Status and Postdivorce Father Involvement With Children », Journal of Family Issues, 23, 5 : 601623.

MARSIGLIO William, AMATO Paul, DAY Randall D., 2000, « Scholarships on fatherhood on 1990s and beyond ", Journal of Marriage and the Family, 62, 4 : 1173-1191.

MARTIAL Agnès, 2003, S'apparenter. Ethnologie des liens de familles recomposées, Paris, Éditions de la Maison des Sciences de l'Homme.

- 2005, « Comment rester liés ? Les comptes des familles recomposées », Terrain, 45 : 67-82.

- 2008, "Changements de nom, changement de filiation », in Fine Agnès (dir.), États 
civils en questions, Paris, éditions du CTHS, coll. « Le regard de l'ethnologue » : 115-138. - 2009a, « Choisir ses héritiers. Recompositions familiales et successions patrimoniales en France et au Québec », Anthropologie et Sociétés, Enfances en péril, 33, $1:$ 193-209.

- 2009b, Le travail parental : du côté des pères séparés et divorcés,

CAF Informations sociales, 4, 154 : 96-104.

MARTIN Claude, 1997, L'après-divorce. Lien familial et vulnérabilité, Rennes, Presses Universitaires de Rennes.

MERLA Laura, 2007, "Père au foyer : une expérience "hors normes", Recherches et prévisions, $90: 17-27$.

NEYRAND Gérard, 2000, L'enfant, la mère et la question du père, Paris, Presses Universitaires de France.

- 2009 [1994], L'enfant face à la séparation des parents. Une solution, la résidence alternée, Paris, Syros.

PRIOUX France, Magali MAZUY et Magali BARBIERI, 2010, « L'évolution démographique récente en France : les adultes vivent moins souvent en couple », Population, 65, 3 : 421474.

QUÉNIART Anne, 1999, «Émancipation ou désancrage social : deux représentations de la rupture parentale chez des pères n'ayant plus de contact avec leur enfant », Déviance et société, 23, $1:$ :91-104.

RÉGNIER-LOILIER Arnaud, 2006, «À quelle fréquence voit-on ses parents ? », Population et Sociétés, INED, 427.

SALAZAR Carles, 2009, "Vérité biologique et fiction sociale dans l'histoire du droit paternel. Essai d'anthropologie juridique ", in Enric Porqueres I Géné (dir.), Défis contemporains de la parenté, Paris, éditions de l'EHESS : 59-80.

SCHWARTZ Olivier, 1990, Le monde privé des ouvriers. Hommes

et femmes du Nord, Paris, Presses Universitaires de France.

THÉRY Irène, 1993, Le démariage, Paris, Odile Jacob.

- 2007, La distinction de sexe, une nouvelle approche de l'égalité,

Paris, Odile Jacob.

THÉRY Irène et Marie-Josèphe DHAVERNAS, 1991, Le beau-parent dans les familles recomposées. Rôle familial, statut social, statut juridique, Paris, Rapport de recherches pour la CNAF.

TRINDER Liz, Mary BEEK and Jo CONNOLLY, 2002, Making Contact : How Parents and Children Negociate and Experience Contact after Divorce, York, Joseph Rowntree Foundation.

VERJUS Anne et Marie VOGEL, 2009, « Le travail parental : un travail comme un autre ?», Informations sociales, CNAF, 4, 154 : 4-6.

VILLENEUVE-GOKALP Catherine, 1994, "Situations et biographies familiales des enfants ", in Henry Léridon et Catherine Villeneuve-Gokalp, Constances et inconstances de la famille, biographies familiales des couples et des enfants, Travaux et Documents, 134, Paris, Presses Universitaires de France, INED : 197-231.

- 1999, «La double famille des enfants de parents séparés », Population, 1 : 9-36.

VIVAS Émilie, 2007, « La séparation des parents ou le décès de la mère distend les relations avec le père », Insee Première, 1157, INSEE : 1-4.

WEBER Florence, 2005, Le sang, le nom, le quotidien : une analyse de la parenté pratique, Paris, Aux Lieux d'Être. 